\section{Extravasation of antineoplastic agents: prevention and treatments}

\author{
Rita Boschi,1 Elena Rostagno2 \\ 1IOR, Bologna; 2Ospedale Sant'Orsola \\ Malpighi, Bologna, Italy
}

\begin{abstract}
The extravasation of antineoplastic agents is an unwanted and distressing situation that can easily occur. It may cause severe and irreversible local injuries. Left untreated, vesicant chemotherapy extravasation can potentially cause tissue necrosis, functional impairment and permanent disfigurement. This article provides a review of current literature regarding recommendations on the prevention and treatment of extravasation of antineoplastic agents.
\end{abstract}

\section{Introduction}

The extravasation of antineoplastic agents is an unwanted and distressing situation that can easily occur. It may cause severe and irreversible local injuries. Extravasation is the inadvertent leakage or escape of a drug or solution from a vein or the unintentional injection into surrounding healthy tissue. It can result in significant tissue damage. Extravasation injury accounts for $0.1-6.5 \%$ of adverse effects related to chemotherapeutic administration. 1,2

Based on their potential to cause local tissue injury, drugs are classified as vesicant, irritant or non-vesicant (Table 1). ${ }^{3-5}$ Vesicant drugs can induce the formation of blisters and/or destroy tissue. Irritant drugs can cause pain at the injection site or along the vein with or without an inflammatory reaction. Some of these agents can potentially cause soft tissue ulcers. This, however, only occurs if a large amount of concentrated drug solution is inadvertently extravasated. Non-vesicant drugs, if extravasated, rarely produce acute reactions or tissue necrosis.

Extravasation can cause tissue damage in different ways: i) the drug is absorbed by local cells in the tissue and binds to critical structures (e.g. DNA, microtubules), causing cell death. After the endocytolysis, surrounding cells can also die through the release of the drug from nearby dead cells. The repetitive nature of this process impairs healing and may result in progressive and chronic tissue injury; 3,6-8 ii) the drug that does not bind to cellular DNA may metabolize and be cleared, limiting the degree of tissue injury. This is, therefore, more easily neutralized. 2,3

\section{Risk factors}

Risk factors for vesicant chemotherapy extravasation have been identified, and patients are at high risk for extravasation when multiple risk factors are present.2,4,5 When considering a treatment regime that contains one or more vesicant drugs, the heath care team should consider the planned duration of treatment and review individual patient characteristics, such as the availability of peripheral veins. The possible placement of a central venous access device should also be evaluated. 4

Risk factors for extravasation from peripheral veins include the presence of small and/or fragile veins, obesity, multiple previous venipunctures, presence of disseminated skin diseases (e.g. eczema or psoriasis), patient movement, and prior treatment. 4 Sensory deficits, altered mental status, somnolence, age (in particular, young patients) that impair the patient's ability to detect a change in sensation at the site of chemotherapy administration increase the risk that he or she may not notice that an extravasation is taking place.2,4,5,9 Additional risk factors include the competence and experience of the person responsible for inserting the intravenous (i.v.) catheter. The risk of extravasation is increased when vesicants are administered via rigid intravenous devices (such as butterfly needles) because these can easily puncture the vein when the patient moves. Also, inadequately secured peripheral i.v. catheters, and catheters with wet or loose dressing, could back out of the vein. When the tip of the catheter is no longer in the vein and a vesicant is administered, an extravasation will occur.2,4,5 Extravasation from central venous catheter (CVC) can occur with needle displacement from an implanted venous access port, mechanical occlusion and subsequent CVC damage, catheter migration, or fibrin sleeve formation and thrombosis.

\section{Signs and symptoms}

It is essential to recognize that an extravasation has taken place as quickly as possible. Signs and symptoms of vesicant extravasation include swelling, redness and/or discomfort that is often described as a burning or stinging sensation. Resistance during drug administration, a slow and sluggish infusion, and lack or loss of a blood return from the i.v. cannula, implanted port or other central venous access device could all be signs of vesicant extravasation.
Correspondence: Rita Boschi,

IOR, via G.C.Pupilli n. 1 - 40136, Bologna, Italy.

E-mail: rboschi@ior.it

Key words: antineoplastic agents, extravasion, pediatrics.

Received for publication: 13 October 2011. Accepted for publication: 2 November 2011.

This work is licensed under a Creative Commons Attribution NonCommercial 3.0 License (CC BYNC 3.0).

CC Copyright R. Boschi and E. Rostagno, 2012

Licensee PAGEPress, Italy

Pediatric Reports 2012; 4:e28

doi:10.4081/pr.2012.e28

\section{Recommendations}

Recommendations have been classified according to the IDSA-United States Public Health Service grading system for ranking recommendations (Table 2).

\section{Recommendations for prevention}

Some precautionary steps to prevent extravasation should be taken.

- Administration of vesicant agents should be carried out through a central line whenever possible, especially if continuous infusion is required (AIII). ${ }^{3-5,10-12}$

- Avoid joints and limbs with impaired arterial, venous, or lymphatic circulation. Optimal vein selection for peripheral vesicant administration is important. Veins that are small and fragile, veins in areas of flexion and veins in arms with lymphedema, or neurological impairment are all situations at risk and administration should be avoided in these cases. If possible, i.v. access should be completed with minimal trauma and repeated attempts should be avoided. When initial efforts are unsuccessful, an alternative vein should be selected, preferably in the opposite arm (AIII) $1-5,11,12$

- Procedures for administering vesicants and managing extravasations should be standardized (AIII). ${ }^{2,10,13}$

- All staff members involved in chemotherapy administration should receive adequate training (AIII).2,5,10,13-15

- Patients should be educated about the risk of vesicant extravasation. Patients should be asked to report any sensation of pain or burning immediately (AIII). $3,5,10,13,14,16$

- The plastic cannula (AIII) with the smallestgauge possible, $4,5,10,16$ and an appropriate needle length for the access device (Port) should be used (AIII).4,5,10,14

- Cannulas and non-coring needles should be secured and insertion sites should be visi- 
ble. During vesicant administration, the site should be monitored for swelling, redness, pain or other symptoms (AIII) $2-5,16$

- A peripheral i.v. catheter must first be tested with i.v. fluids at a high flow to determine the patency of the vessel to be used and exclude extravasation prior to the administration of chemotherapy drugs (AIII).2,3,16

- A blood return should always be obtained before vesicants are administered (AIII). 2,3,5,14,16

- Peripheral vesicants are administered by gravity infusions or i.v. bolus and should not be infused using an infusion pump since the pump may continue to deliver a vesicant into the tissue until the pump alarm is triggered (AIII).5,11,16

- The vein should be flushed with i.v. fluids every 2-3 min during bolus injection of the chemotherapeutic agent and should be wellflushed again at the end of administration.2,11,12

- If more drugs must be administered, vesicants should be administered first because veins will not have been irritated by other agents and because post-vesicant flushing will preserve venous integrity (BIII). 5,12

\section{Recommendations for treatment}

When an extravasation occurs or is suspected, the first action to take is to stop the infusion (AII). ${ }^{2-4,10,16}$ Leave the cannula in position and attempt to aspirate as much of the vesicant as possible from the device; if an antidote is available, an appropriate amount should be instillated through the existing i.v. (AIII).2,3,10,17,18

Leave the non-coring port needle in place and attempt to aspirate as much of the vesicant as possible from the device; if an antidote is available, an appropriate amount should be instillated through the existing i.v. (AIII).2,3,10,17,18

Administrate specific antidotes as indicated (Table 3). Antidotes should be given within one hour through the needle and/or subcutaneously (s.c.) around the site of extravasation (AIII). $2,3,10,14,16-18$

Rest and elevate the affected site for $48 \mathrm{~h}$ to promote the normal absorption and drainage of loculated extravasated fluids (BIII).2,3

Allow local cooling or warming for at least 15-30 min four times a day for 24-48 h (Table 3) (BIII). ${ }^{2-4,10,14,16}$

Document the extravasation as required and, if considered necessary, take a photograph of the affected site (AIII). ${ }^{2-5,10,14,15} \mathrm{An}$ extravasation kit may be useful (AIII).10,18

The site should be closely monitored at $24 \mathrm{~h}$, 1 week, 2 weeks, and as necessary for pain, redness, swelling, ulceration, or necrosis, depending on the extent of tissue damage (AIII).11,14,15

\section{Antidotes}

Antidotes that are considered useful and safe are outlined below along with their mechanisms of action:

i) Hyaluronidase degrades hyaluronic acid, breaks down subcutaneous tissue bonds promoting drug diffusion through the interstitial space and enhances the absorption of injected substances. ${ }^{1,14,17}$ Administer $1 \mathrm{~mL}$ of the hyaluronidase solution in five $0.2 \mathrm{~mL}$ injections into the extravasation site using a 25 gauge or smaller needle, changing the needle for each injection. ${ }^{4,14,17}$ ii) Sodium thiosulfate neutralizes the vesicant by creating an alkaline-rich site, to which alkylating agents have an affinity. The vesicant binds to the sodium thiosulfate instead of the tissue and the by-product is excreted in the urine.1,17 To prepare a 1/6 molar solution, mix $4 \mathrm{~mL}$ of $10 \%$ sodium thiosulfate with $6 \mathrm{~mL}$ of sterile water for injection. Inject the solution into the extravasation site using a 25 gauge or smaller needle, changing the needle for each injection. $4,14,17$

iii) DMSO is a common solvent capable of swiftly penetrating tissue. The primary mechanism of action is the drug's ability to neutralize free-radical accumulation and reduce tis-

Table 1. Vesicant and irritant chemotherapeutic agents.

\begin{tabular}{lll}
$\begin{array}{l}\text { DNA-binding vesicant } \\
\text { drugs }\end{array}$ & $\begin{array}{l}\text { Alkylating agents } \\
\text { Anthracyclines } \\
\text { Antitumor antibiotics }\end{array}$ & $\begin{array}{l}\text { Mechloretamine } \\
\text { Doxorubicin, Daunorubicin, Epirubicin, Idarubicin } \\
\text { Mitomycin, Dactinomycin, Mitoxantrone* }\end{array}$ \\
$\begin{array}{ll}\text { Non-DNA binding } \\
\text { vesicant drugs }\end{array}$ & $\begin{array}{l}\text { Vinca alkaloids } \\
\text { Taxane }\end{array}$ & $\begin{array}{l}\text { Vinblastine, Vincristine, Vinorelbine, Vindesine } \\
\text { Paclitaxel, Docetaxel }\end{array}$ \\
\hline Irritant drugs & Alkylating agents & Carmustine, Dacarbazine, Ifosfamide, Melphalan, \\
& & Thiotepa, \\
& Platinum analogs & Carboplatin, ${ }^{\circ}$ Cisplatin, ${ }^{\sharp}$ Oxaliplatin \\
& Topoisomerase II inhibitors & Teniposide, Etoposide \\
& Anthracyclines & Liposomal Doxorubicin, Liposomal Daunorubicin \\
& Topoisomerase I inhibitors & Irinotecan, Topotecan \\
\hline
\end{tabular}

*Vesicant property depending on concentration. 'Usually classified as irritants but reported to be mild vesicants. \#Reported as a vesicant if more than $20 \mathrm{~mL}$ of $0.5 \mathrm{mg} / \mathrm{mL}$ concentration extravasated.

Table 2. Classification of evidence.

Quality of evidence recommendation

Table 3. Antidotes and treatments.

\begin{tabular}{lcc}
\hline Extravasated vesicant & Antidote & Treatment \\
Anthracyclines & $\begin{array}{c}\text { DMSO } \\
\text { Dexrazoxane } \\
\text { Cisplatin }\end{array}$ & Topical cooling \\
\hline Dactinomycin & Sodium thiosulfate & Topical cooling \\
Docetaxel & Hyaluronidase & Topical cooling \\
\hline Mechloretamine & Sodium thiosulfate & Topical cooling \\
Mytomicin & DMSO & Topical cooling \\
\hline Mitoxantrone & DMSO & Topical cooling \\
Oxaliplatin & Sodium thiosulfate & Topical warming \\
\hline Paclitaxel & & Topical cooling \\
Vinca alkaloids & Hyaluronidase & Topical warming
\end{tabular}

Evidence from $>1$ correctly randomized, controlled trial Evidence from: > lwell-designed non-randomized clinical trials; cohort or case-controlled analytical studies (preferably from $>1$ center); multiple time-series; or results that raise concerns from uncontrolled experiments Evidence from opinions of respected authorities, based on clinical experience, descriptive studies, or reports of expert committees.

Good evidence to support a recommendation for use Moderate evidence to support a recommendation for use Poor evidence to support a recommendation 
sue damage by enhancing systemic absorption of the extravasated drug. It possesses vasodilatatory, analgesic, anti-inflammatory properties and enhances skin permeability. ${ }^{1}$ Apply topically to the skin twice the size of infiltration with a cotton swab and allow it to dry (do not cover), repeating every 6-8 $\mathrm{h}$ for 7-14 days. ${ }^{3}$

iv) Dexrazoxane: is the only treatment that has received European Commission authorization and US Food and Drug Administration approval for anthracycline extravasation treatment. The drug binds to iron and prevents the formation of free radicals that are thought to play a major role in the development of extravasation-induced tissue necrosis.4,14,17,19,20 Administer intravenously in a vein in an area away from the extravasation site. Infuse 1,000 $\mathrm{mg} / \mathrm{m}^{2}$ within $6 \mathrm{~h}$ of extravasation on Day 1 , $1,000 \mathrm{mg} / \mathrm{m}^{2}$ on Day 2 , and $500 \mathrm{mg} / \mathrm{m}^{2}$ on Day 3 . Maximum daily dose is $2,000 \mathrm{mg} .4,14,17$

\section{Topical heat or cooling}

The benefits of thermal treatment to reduce the damage caused by the extravasation of antineoplastic drugs have been confirmed in animal studies. However, literature data remain controversial.1,21 The application of cold compresses is based on the induction of vasoconstriction with a consequent decrease in the diffusion velocity of the drug within the tissues, thus reducing the area of potential tissue damage. The mechanism of action of heat applied to the site of extravasation is to induce vasodilatation and, consequently, to facilitate the increase in absorption and systemic distribution of the cytostatic drug.

\section{References}

1. Kassner E. Evaluation and treatment of chemotherapy extravasation injuries. J Pediatr Oncol Nurs 2000;17:135-48.

2. Goolsby TV, Lombardo FA. Extravasation of chemotherapeutic agents: prevention and treatment. Semin Oncol 2006;33:13943.

3. Ener RA, Meglathery SB, Styler M. Extravasation of systemic hemato-oncological therapies. Ann Oncol 2004;15:85862.

4. Schulmeister L. Extravasation management: clinical update. Semin Oncol Nurs 2011;27:82-90.

5. Sauerland C, Engelking C, Wickham R, Corbi D. Vescicant extravasation part I: mechamisms, pathogenesis, and nursing care to reduce risk. Oncology Nursing Forum 2006;33:1134-41.

6. Polovich M, White J, Kelleher L. Chemotherapy and biotherapy guidelines and recommendations for practice. Pittsburgh, PA: Oncology Nursing Society; 2005

7. Dorr RT, Dorland MS, Koenig LM, et al. High levels doxorubicin in the tissues of a patient experiencing extravasation during a 4-day infusion. Cancer 1989; 64:2462-9.

8. Sonneveld P, Wassenaar HA, Nooter K. Long persistence of doxorubicin in human skin after extravasation. Cancer Treat Rep 1984;68:895-6.

9. Doellman D, Hadaway L, Bowe-Geddes LA, et al. Infiltration and extravasation: update on prevention and management. Journal of Infusion Nursing 2009;32:20311.

10. Wengstrom Y, Margulies A. European Oncology Nursing Society extravasation guidelines. European Journal of Oncology Nursing 2008;12:357-61.

11. Mullin S, Beckwith M, Tyler L. Prevention and management of antineoplastic extravasation injury. Hospital Pharmacy 2000;35:57-74.
12. How C, Brown J. Extravasation of cytotoxic chemotherapy fron peripheral veins. European Journal of Oncology Nursing 1998;2:51-8.

13. Jones L, Coe P. Extravasation. European Journal of Oncology Nursing 2004;8:3558.

14. Schulmeister L. Managing vescicant extravasation. The Oncologist 2008;13: 284-8.

15. Adami NP, de Gutièrrez MG, da Fonseca SM, de Almeida EP. Risk management of extravasation of cytostatic drugs at the Adult Chemotherapy Outpatient Clinic of a university hospital. J Clin Nurs 2005;14: 876-8.

16. Schulmeister L. Preventing and managing vescicant chemotherapy extravasation. The Journal of Supportive Oncology 2010; 8:212-5.

17. Schulmeister L. Vesicant chemotherapy extravasation: antidotes and treatments. Clinical Journal of Oncology Nursing 2009;13:395-8.

18. Wickham R, Engelking C, Sauerland C, Corbi D. Vescicant extravasation part II: evidence-based management and continuing controversies. Oncology Nursing Forum 2006;33:1143-50.

19. Langstein HN, Duman H, Feeling D, et al. Retrospective study of management of chemotherapeutic extravasation injury. Ann Plas Surg 2002;49:369-74.

20. Bos AM, Van der Graaf WT, Willemse PH. A new conservative approach to extravasation of anthracyclines with dimethylsulfoxide and dexrazoxane. Acta Oncol 2001;40:541-42

21. Albanell J, Baselga J. Systematic therapy emergencies. Semin Oncol 2000;27:34761 . 